\title{
BOUNDED $J$-FRACTIONS
}

H. S. WALL

1. Introduction. A $J$-fraction

$$
\frac{1}{b_{1}+z-\frac{a_{1}^{2}}{b_{2}+z-\frac{a_{2}^{2}}{b_{3}+z-.}}}
$$

in which the coefficients $a_{p}$ and $b_{p}$ are complex constants and $z$ is a complex parameter, is said to be bounded if there exists a constant $M$ such that

$$
\left|a_{p}\right| \leqq M / 3, \quad\left|b_{p}\right| \leqq M / 3, \quad p=1,2,3, \cdots .
$$

This condition can be formulated in terms of $J$-forms in accordance with the following theorem.

THEOREM 1.1. The J-fraction (1.1) is bounded if and only if there exists a constant $N$ such that

$$
\begin{aligned}
& \left|\sum_{p=1}^{n} b_{p} u_{p} v_{p}-\sum_{p=1}^{n-1} a_{p}\left(u_{p} v_{p+1}+u_{p+1} v_{p}\right)\right| \\
& \leqq N\left(\sum_{p=1}^{n}\left|u_{p}\right|^{2} \cdot \sum_{p=1}^{n}\left|v_{p}\right|^{2}\right)^{1 / 2}, \quad n=1,2,3, \cdots,
\end{aligned}
$$

for all values of the variables $u_{p}$ and $v_{p}$, the constant $N$ being independent of the variables and of $n$.

In fact, if (1.3) holds then we find, on specializing the values of the $u_{p}$ and $v_{p}$, that $\left|b_{p}\right| \leqq N,\left|a_{p}\right| \leqq N, p=1,2,3, \cdots$; and if (1.2) holds then, by Schwarz's inequality, (1.3) holds with $N=M$.

If (1.3) holds, then the $J$-form $\sum b_{p} u_{p} v_{p}-\sum a_{p}\left(u_{p} v_{p+1}+u_{p+1} v_{p}\right)$ is said to be bounded, and the least value of $N$ which can be used in that inequality is called the norm of the $J$-form. We shall also call this number the norm of the $J$-fraction. When (1.2) holds then, as pointed out above, (1.3) holds with $N=M$. Hence the norm of the $J$-fraction does not exceed the least number $M$ which can be used in (1.2).

Presented to the Society, April 27, 1946; received by the editors February 28, 1946. 
THEOREM 1.2. If (1.2) holds, then the J-fraction converges uniformly for $|z| \geqq M$.

For if the $J$-fraction is transformed by an equivalence transformation so that all the partial denominators are equal to unity, then the $n$th partial numerator is

$$
-\frac{a_{n-1}^{2}}{\left(b_{n-1}+z\right)\left(b_{n}+z\right)} .
$$

If $|z| \geqq M$, and (1.2) holds, this has modulus not greater than $1 / 4$. Hence it follows by a well known theorem that the $J$-fraction converges uniformly for $|z| \geqq M$.

In the case of the $J$-fraction

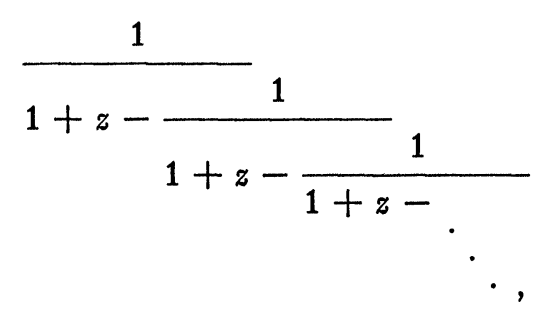

the least number $M$ which can be used in (1.2) is $M=3$. Hence the $J$-fraction converges uniformly for $|z| \geqq 3$. It diverges if $z$ is real, positive, and less than 3 . On the other hand, for the $J$-fraction

$$
\frac{1}{z-\frac{(1 / 4)}{z-\frac{(1 / 4)}{z-} \cdot},}
$$

the least value of $M$ which can be used in (1.2) is $M=3 / 2$. The norm of this $J$-fraction is $N=1$, and it converges for $|z| \geqq 1$. In fact, it converges if $z$ is not on the real interval $-1<x<+1$.

The principal object of this note is to show that a $J$-fraction with norm $N$ converges if $z$ is not in a certain convex set contained in the circle $|z|=N$. Moreover, if the partial numerators $a_{p}{ }^{2}$ are different from zero, the corresponding $J$-matrix has a unique bounded reciprocal for all $z$ not in this convex set. It was shown by Hellinger and Toeplitz [3] $]^{1}$ that there is a unique bounded reciprocal for $|z|>N$.

${ }^{1}$ Numbers in brackets refer to the Bibliography at the end of the paper. 
2. Convergence of bounded $J$-fractions. Let $a=e^{i \theta}$ be a complex number with modulus unity. Then the $J$-fractions (1.1) and

$$
\frac{a}{b_{1} a+Z-\frac{\left(a_{1} a\right)^{2}}{b_{2} a+Z-\frac{\left(a_{2} a\right)^{2}}{b_{3} a+Z-}}} \quad Z=a z
$$

are equivalent in the sense that their $n$th approximants are identical with one another for $n=1,2,3, \cdots$. Also, they obviously have one and the same norm.

Let

$$
\alpha_{p}(\theta)=I\left(a_{p} a\right), \quad \beta_{p}(\theta)=I\left(b_{p} a\right), \quad p=1,2,3, \cdots .
$$

Then, if (1.1) is bounded, it follows from (1.3) that there exists a finite constant $Y(\theta)$ such that

$$
\sum_{p=1}^{n}\left[\beta_{p}(\theta)+Y(\theta)\right] x_{p}^{2}-2 \sum_{p=1}^{n-1} \alpha_{p}(\theta) x_{p} x_{p+1} \geqq 0, n=1,2,3, \cdots,
$$

for all real values of $x_{1}, x_{2}, x_{3}, \cdots$. If $Y_{0}(\theta)$ is the least value of $Y(\theta)$ which can be used in (2.3), then we must have

$$
\left|Y_{0}(\theta)\right| \leqq N, \quad 0 \leqq \theta<2 \pi,
$$

where $N$ is the norm of the $J$-fraction.

From (2.3) it follows that if we put $Z=i Y(\theta)+\zeta$ in (2.1), then (2.1) is a positive definite $J$-fraction in the variable $\zeta$. Therefore, if $c$ is a positive constant, the $n$th approximant of (1.1), which is the same as the $n$th approximant of $(2.1)$, satisfies the inequality [1]

$$
\left|\frac{A_{n}(z)}{B_{n}(z)}\right| \leqq \frac{1}{c},
$$

provided $I(\zeta) \geqq c$, that is, provided

$$
x \sin \theta+y \cos \theta \geqq Y(\theta)+c, \quad \text { where } z=x+i y ;
$$

and $B_{n}(z) \neq 0$ when (2.4) holds. This can be interpreted geometrically as follows. Let $K$ denote the set of all points $z=x+i y$ such that

$$
x \sin \theta+y \cos \theta \leqq Y(\theta) \quad \text { for } 0 \leqq \theta<2 \pi .
$$


Then, $K$ is a convex set of which the straight lines $x \sin \theta+y \cos \theta$ $=Y(\theta)$ are the supporting lines; the zeros of all the denominators $B_{n}(z)$ of the $J$-fraction (1.1) are in $K$; the approximants of the $J$-fraction are uniformly bounded over any domain whose distance from $K$ is positive. We shall let $K_{0}$ denote the convex set determined in this way corresponding to the function $Y_{0}(\theta)$ defined above.

By Theorem 1.2, the $J$-fraction converges if $|z|$ is sufficiently large. We may then conclude immediately by a theorem of Stieltjes [6] that the following theorem is true.

THEOREM 2.1. A bounded J-fraction converges uniformly over every bounded closed region whose distance from the convex set $K_{0}$ is positive. In particular, the J-fraction converges if $|z|>N$, where $N$ is the norm of the J-fraction.

We note the following special cases. If the coefficients $a_{p}$ and $b_{p}$ are all real, then $Y_{0}(0)=Y_{0}(\pi)=0$, so that $K_{0}$ reduces to an interval of the real axis contained in the interval $-N \leqq x \leqq+N$. If the $a_{p}$ are pure imaginary and the $b_{p}$ are real and positive, then the set $K_{0}$ is contained in the left half-plane, $x=R(z) \leqq 0$.

3. Bounds for the zeros of a polynomial. The preceding considerations furnish a method for determining bounds for the zeros of a polynomial. Let $P(z)$ be a polynomial of degree $n, n>1$, and let $Q(z)$ be any polynomial of degree $n-1$ such that there is a continued fraction expansion of the form

$$
\begin{aligned}
\frac{Q(z)}{P(z)}=\frac{c}{b_{1}+z-\frac{a_{1}^{2}}{b_{2}+z-}} & \\
\ddots & -\frac{a_{n-1}^{2}}{b_{n}+z},
\end{aligned}
$$

where $a_{p} \neq 0, p=1,2,3, \cdots, n-1$, and $c \neq 0$. This expansion can be easily obtained by applying the euclidean algorithm for the greatest common divisor to $Q(z)$ and $P(z)$. Let $K_{0}$ be the convex set which is associated with this $J$-fraction in the manner indicated in $\S 2$. Then the zeros of $P(z)$ are all contained in $K_{0}$.

One may readily obtain a rectangle containing the set $K_{0}$. In fact, if we use the notation of $\S 2$, such a rectangle is given by

$$
\begin{array}{ll}
y \leqq Y(0), & x \leqq Y(\pi / 2), \\
y \geqq-Y(\pi), & x \geqq-Y(3 \pi / 2) .
\end{array}
$$


This rectangle is obtained by minimizing four real quadratic forms.

By way of illustration, let $P(z)=z^{3}+(2+i) z^{2}+(3+i) z+(2 i+2)$, and take $Q(z)=2 z^{2}+i z+2$. Then,

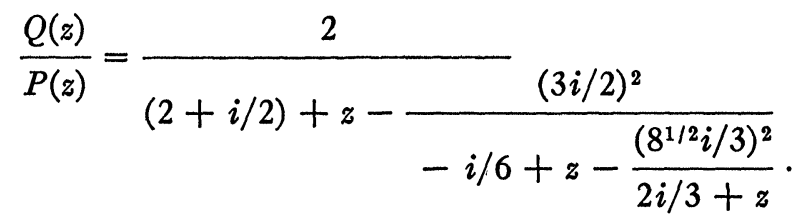

We require lower bounds $-Y(\theta)$ for the quadratic form

$(2 \sin \theta+(1 / 2) \cos \theta) x_{1}^{2}-(1 / 6) \cos \theta x_{2}^{2}+(2 / 3) \cos \theta x_{3}^{2}$

$$
-3 \cos \theta x_{1} x_{2}-\left(2^{5 / 2} / 3\right) \cos \theta x_{2} x_{3},
$$

under the condition $x_{1}{ }^{2}+x_{2}{ }^{2}+x_{3}{ }^{2}=1$, and for $\theta=0, \pi / 2, \pi, 3 \pi / 2$. Easily determined lower bounds are given by

$$
Y(0)=19 / 6, \quad Y(\pi / 2)=0, \quad Y(\pi)=11 / 3, \quad Y(3 \pi / 2)=2 .
$$

Therefore, the zeros of $P(z)$ are contained in the rectangle

$$
\begin{array}{ll}
y \leqq 19 / 6, & x \leqq 0, \\
y \geqq-11 / 3, & x \geqq-2 .
\end{array}
$$

The zeros of $P(z)$ are actually equal to

$$
-1-i, \quad \frac{-1-7^{1 / 2} i}{2}, \quad \frac{-1+7^{1 / 2} i}{2} .
$$

The size of the rectangle depends upon the choice of the polynomial $Q(z)$. In fact, it is easy to show that the zeros of $Q(z)$ also lie in the convex set $K_{0}$. Furthermore, the computational difficulties are less for some choices of $Q(z)$ than they are for other choices. Let

$$
P(z)=z^{n}+\left(p_{1}+i q_{1}\right) z^{n-1}+\left(p_{2}+i q_{2}\right) z^{n-2}+\cdots+\left(p_{n}+i q_{n}\right) .
$$

Then, if

$$
Q(z)=p_{1} z^{n-1}+i q_{2} z^{n-2}+p_{3} z^{n-3}+i q_{4} z^{n-4}+\cdots,
$$

the computation involved in obtaining the $J$-fraction expansion for $Q(z) / P(z)$ is especially simple. Moreover, from this expansion one can determine immediately the number of zeros of $P(z)$ in each of the half-planes $R(z)<0$ and $R(z)>0$. For details, we refer the reader to a recent paper of Frank [2].

4. The bounded reciprocal of a bounded $J$-matrix. We suppose 
that (1.1) is bounded, that $a_{p} \neq 0, p=1,2,3, \cdots$, and consider the $J$-matrix

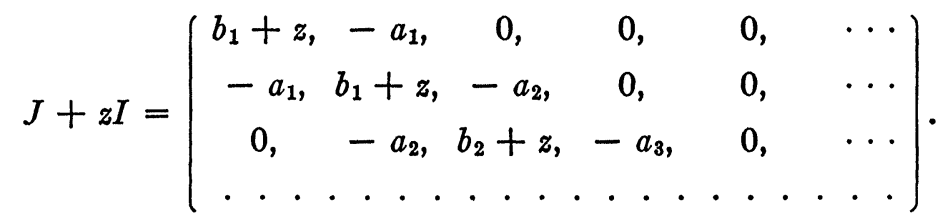

If the norm of (1.1) is $N$, and $|z|>N$, then the matrix $J+z I$ has a unique bounded reciprocal which is given by

$$
(J+z I)^{-1}=\frac{I}{z}-\frac{J}{z^{2}}+\frac{J^{2}}{z^{3}}-\cdots .
$$

This is a matrix whose elements are power series in $1 / z$, convergent for $|z|>N$. In particular, the element in the first row and first column is the power series expansion of the $J$-fraction, and its sum is the value of the $J$-fraction (Hellinger and Toeplitz [3]).

We can now show that $J+z I$ has a unique bounded reciprocal for any $z$ not in the set $K_{0}$ defined in $\$ 2$. In fact, if we put $Z=i Y_{0}(\theta)+\zeta$ in (2.1), then, as we have seen, the $J$-fraction is a positive definite $J$-fraction in the variable $\zeta$. The corresponding $J$-matrix is

$$
a J+i Y_{0}(\theta) I+\zeta I \text {. }
$$

Inasmuch as the series $\sum\left(1 /\left|a a_{p}\right|\right)$ is divergent, the determinate case holds for the $J$-fraction [1] and consequently [7] the matrix (4.2) has a unique bounded reciprocal for $I(\zeta)>0$. We therefore conclude immediately that the $J$-matrix $J+z I$ has a unique bounded reciprocal for any $z$ not in the set $K_{0}$ defined in $\$ 2$.

5. Functions represented by $J$-fractions. Every infinite subsequence of approximants of a positive definite $J$-fraction contains an infinite subsequence which converges for $I(z)>0$ to a function which is analytic and has a negative imaginary part in this domain, and which has the form

$$
f(z)=\int_{-\infty}^{+\infty} \frac{d \phi(u)}{z-u},
$$

where $\phi(u)$ is bounded and nondecreasing. There are functions which are analytic and have negative imaginary parts for $I(z)>0$ which are not limits of sequences of approximants of positive $J$-fractions. In fact, the most general function of this description has the form 


$$
\begin{aligned}
& \int_{-\infty}^{+\infty} \frac{1+z u}{z-u} d \phi(u)+a-b z \\
& \quad=\int_{-\infty}^{+\infty}\left(\frac{u}{1+u^{2}}+\frac{1}{z-u}\right)\left(1+u^{2}\right) d \phi(u)+a-b z
\end{aligned}
$$

where $a$ and $b$ are real, $b \geqq 0$, and $\phi(u)$ is bounded and nondecreasing. This can be seen as follows. F. Riesz [5] and Herglotz [4] showed that a function $f(w)$ is analytic and has a positive real part for $|w|<1$ if and only if it has the form

$$
f(w)=\int_{0}^{2 \pi} \frac{e^{i t}+w}{e^{i t}-w} d \sigma(t)+i a,
$$

where $a$ is real and $\sigma(t)$ is bounded and nondecreasing. If we multiply this integral by $-i$ and make the substitution

$$
w=\frac{1+i z}{1-i z},
$$

mapping the unit circle upon the upper half-plane, we obtain after simple transformations

$$
\int_{0}^{2 \pi} \frac{1-z \tan (t / 2)}{\tan (t / 2)+z} d \sigma(t)+a .
$$

This can be transformed into (5.2) if we put $u=\tan (t / 2)$.

We take this occasion to point out that there exists an identical continued fraction transformation of the integral (5.2). We have the following theorem.

THEOREM 5.1. A necessary and sufficient condition for a function to be analytic and have a negative imaginary part for $I(z)>0$ is that it have a continued fraction expansion of the form

$$
\begin{aligned}
& \frac{c}{z-r_{0}-\frac{g_{1}\left(1+z^{2}\right)}{z-r_{1}-\frac{\left(1-g_{1}\right) g_{2}}{\left(1+z^{2}\right)}}} \\
& z-r_{2}-\frac{\left(1-g_{2}\right) g_{3}}{z-r_{3}-}\left(1+z^{2}\right)
\end{aligned}
$$

where $c>0,0<g_{p}<1,-\infty<r_{p-1}<+\infty, p=1,2,3, \cdots$, or a terminat- 
ing continued fraction expansion of this form in which the last $g_{p}$ which appears may be equal to unity. The continued fraction converges uniformly over every bounded closed region within the half-plane $I(z)>0$, and is uniquely determined by the function expanded.

To prove this, it is only necessary to make the substitution

$$
z=\frac{4 w}{(1-w)^{2}}
$$

in the continued fraction (3.14) of [8], multiply the resulting continued fraction by $-i$, and then make the substitution (5.3) above.

\section{BIBLIOGRAPHY}

1. J. J. Dennis and H.S. Wall, The limit-circle case for a positive definite J-fraction, Duke Math. J. vol. 12 (1945) pp. 255-273.

2. Evelyn Frank, On the zeros of polynomials with complex coefficients, Bull. Amer. Math. Soc. vol. 52 (1946) pp. 144-157.

3. E. Hellinger and O. Toeplitz, Zur Einordnung der Kettenbruchtheorie in die Theorie der quadratischen Formen von unendlichvielen Veränderlichen, Journal für Mathematik vol. 14 (1914) pp. 212-238.

4. G. Herglotz, Über Potenzreihen mit positivem, reellem Theil im Einheitskreis, Berichte über die verhandlungen der K. Sächsische Akademie der wissenschaften zu Leipzig vol. 63 (1911) pp. 501-511.

5. F. Riesz, Sur certains systems singuliêrs d'equations intègrales, Ann. École Norm. (3) vol. 28 (1911) pp. 34-62.

6. T. J. Stieltjes, Euvres, vol. 2, p. $451 \mathrm{ff}$.

7. H. S. Wall, Reciprocals of J-matrices, Bull. Amer. Math. Soc. vol. 52 (1946) pp. 680-685.

8. - Continued fraction expansions for functions with positive real parts, Bull. Amer. Math. Soc. vol. 52 (1946) pp. 138-143.

ILLINOIS InstituTE of TEChNOLOGY 